\title{
Hypospadias: an algorithm for repair with the aid of the microscope in 102 patients
}

\author{
Mohamed Maher ${ }^{1}$, Maria Chasapi ${ }^{2}$, Milind Dalal ${ }^{2}$ \\ ${ }^{1}$ University Hospitals Birmingham NHS Foundation Trust, Queen Elizabeth Hospital, B15 2TH Birmingham, UK. \\ ${ }^{2}$ Lancachire Teaching Hospitals NHS Foundation Trust, Royal Preston Hospital, PR2 9HT Preston, UK.
}

Correspondence to: Mr. Mohamed Maher, University Hospitals Birmingham NHS Foundation Trust, Queen Elizabeth Hospital, B15 2TH Birmingham, UK. E-mail: mohamed.maher@doctors.org.uk

How to cite this article: Maher M, Chasapi M, Dalal M. Hypospadias: an algorithm for repair with the aid of the microscope in 102 patients. Plast Aesthet Res 2017;4:64-9.

\section{Article history: \\ Received: 10-12-2016 \\ Accepted: 18-04-2017 \\ Published: 27-04-2017}

Key words:

Hypospadias,

one stage/two stage hypospadias

repairs,

urethrocutaneous fistula,

hypospadias algorithm,

microscope

\begin{abstract}
Aim: To demonstrate an algorithmic approach to hypospadias repair with the aid of the surgical microscope as a teaching aid, as well as an ergonomic tool. Methods: One hundred and two patients were operated on between 2009 and 2013, all by the senior surgeon (M Dalal). This is a retrospective review of results in the case series. The procedures used were one stage repair with no incision of the urethral plate, or the two stage Bracka repair. Results: Fifty-six patients underwent the one stage procedure and 46 patients underwent the two stage Bracka repair. Early complication rate was $4.9 \%(n=5)$ and late complication rate was $10.7 \%$ $(n=11)$. The fistula rate specific to one stage and two stage procedures was $5.3 \%(n=3)$ and $13 \%(n=6)$ respectively. Conclusion: A structured approach to hypospadias surgery will aid the surgeon in choosing the appropriate procedure for the appropriate patient. The authors have chosen to use 2 types of repair for all hypospadias subsets based on the algorithm. They find the microscope an invaluable teaching aid, whilst at the same time providing an ergonomic benefit, and providing a wide range of surgical site magnification.
\end{abstract}

\section{INTRODUCTION}

Hypospadias surgery has been performed for decades and centuries. It has evolved quite rapidly and there have been many procedures described along with their modifications. It remains a relatively common condition, occurring in 1:300 live births. ${ }^{[1]}$ Hypospadias is characterised by the presence of an abnormally sited ventral urethral meatus, a dorsally hooded prepuce, and varying degree of penile ventral curvature "chordee". The main aspect in its diagnosis is the abnormal position of the ventral urethral meatus, and to many, this position is related to complexity of the case and the type of repair chosen; when in fact there are other associated morphological aberrations that should be taken into consideration when considering repair. Common meatal positions are distal penile $70 \%$, mid-penile $10 \%$, and proximal types $20 \%$. $^{[2]} \mathrm{We}$ follow an algorithm, which helps in choice of repair irrespective of the meatal site. The surgical procedure in itself requires magnification and a bloodless field to execute a successful repair.

cc) (7) This is an open access article distributed under the terms of the Creative Commons AttributionBY NC SA NonCommercial-ShareAlike 3.0 License, which allows others to remix, tweak, and build upon the work non-commercially, as long as the author is credited and the new creations are licensed under the identical terms.

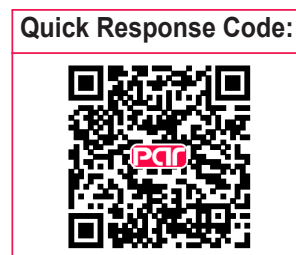




\section{METHODS}

One hundred and two patients were operated upon between 2009 and 2013; the senior author (M. Dalal) performed all patients. Inclusion criteria included primary hypospadias in the paediatric population irrespective of age at the time of initial presentation in clinic. Exclusion criteria, was 1 adult with delayed presentation of primary hypospadias, and 5 cases of phimosis, thought to have concealed hypospadias, treated with circumcision only. The following steps are common to both one stage and two stage procedures. All patients were subjected to a general anaesthetic augmented with a caudal block; for prolonged postoperative pain relief. After induction, co-amoxiclav antibiotic (Augmentin-GlaxoSmithKline) at a dose of $30 \mathrm{mg} / \mathrm{kg}$ is given intravenously over 3-4 min. Medical photography after obtaining the consent from the parents is a routine part of our practice. The photographs are taken in 2 views once the patient is covered in surgical drapes.

The foreskin is retracted and all the smegma removed with a swab soaked in aqueous chlorhexidine, after which the surgeon exchanges the gloves to commence surgery. A urethral dilator size $6 / 8-8 / 10$ is passed after lubrication to ensure that there is an adequate urethral calibre. A polypropylene 5-0 suture (Prolene-Ethicon) is passed on the dorsal surface of the glans for retraction. An 8 French (Fr) urethral catheter is secured at the base of the penis with a haemostat as a tourniquet to facilitate a bloodless field. The microscope (Carl Zeiss Microscopy $\mathrm{GmbH}$ ) microscope is brought into the field at this stage to aid in visualizing the anatomical landmarks as well as carrying out the procedure under $\times 3.5-\times 6$ magnification [Figure 1]. A fine nib quill and ink are used to mark the incision lines, the markings for which differ between the one and two stage procedure. After the finishing the surgery, the suture line is covered soft nonadherent paraffin impregnated gauze (Jelonet-Smith \& Nephew) and sandwiched on the child's abdomen between two layers of low-adhesive perforated plastic films (Melolin-Smith \& Nephew). This is further secured with a broad sheet of adhesive tape (Mefix-Mölnlycke Healthcare). The same adhesive tape is then used to secure the catheter and the paediatric urine bag. This dressing is left undisturbed for 1 week.

Post-operatively patients are kept on oral co-amoxilcav (Augmentin-GlaxoSmithKline) $0.25 \mathrm{~mL} / \mathrm{kg}$ of 125/31 suspension 3 times daily for ages up to 1 year of age and $5 \mathrm{~mL}$ of $125 / 31$ suspension 3 times daily for children older than 1 year. Oxybutynin is administered to prevent bladder spasm, at a dose of $1.25-2.5 \mathrm{mg}$,
3 times a day. Both medications are continued until the catheter is removed at 1 week post-operatively. Analgesics are given as and when required. We aim to discharge patients on the second post-operative day. They are reviewed at 1 and 2 weeks postoperatively. They then are reviewed at 3 months, and from then on yearly till school age.

\section{One stage repair (without urethral plate incision)}

A subcoronal marking is made on the dorsal penile surface and is continued ventrally to the edge of the urethral plate. The ventral markings are made all around the edges of the urethral plate and around the anomalous urethral opening in a "U" shaped design. The ventral incision is made around the urethral plate, and care is taken when dissecting the ventral skin off the anomalous urethral opening, as the corpus spongiosum is deficient, and there is a chance of injury to the urethra. The use of a urethral dilator to guide this part of the dissection can be very helpful [Figure 2]. Ventral chordee encountered at this stage is corrected by degloving the penis. Glans flaps are dissected off the corpora cavernosa with a number fifteen Beaver blade (Beaver Visitec), to achieve a tension free ventral repair over the reconstructed neo-urethra. The dorsum of the penis is degloved in the sub-Dartos plane, and

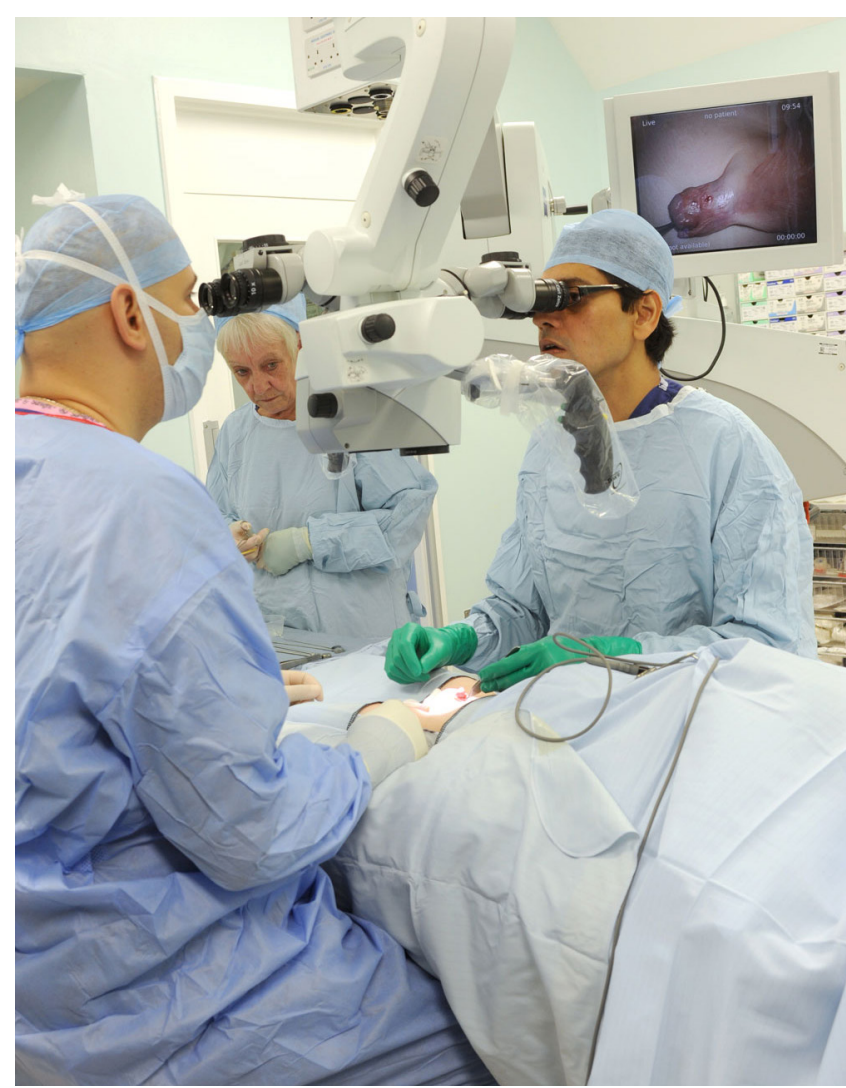

Figure 1: Use of microscope during surgery with the background screen used for intraoperative teaching 
a thin Dartos fascia flap is dissected starting at the distal end of the inner layer of preputal skin. Care is taken to carry the dissection proximally with adequate length in a manner that will allow tension free ventral transposition after neo-urethral reconstruction. This avoids rotation of the penis.

At this stage, a silastic size $6 \mathrm{Fr}$ catheter is passed in the urethra and $1.5 \mathrm{~mL}$ of distilled water is used inflate the balloon. The penis is retracted cephalad to allow for the reconstruction of the neourethra. The first step of the reconstruction is to form the distal most part of the neourethra with a single interrupted polyglactin 7-0 suture (Vicryl-Ethicon) around the inserted catheter. This produces a natural slit like urethral opening. The neourethra is then created starting at the edge of the anomalous ventral meatus and is carried distally with a running submucosal polyglactin 7-0 suture (VicrylEthicon) using the native urethral plate. In contrast with the tabularized incised plate (TIP) procedure described by Snodgrass, the urethral plate was not incised in all of the patients but one. The width of the urethral plate is not routinely measured, but in general, it has to be

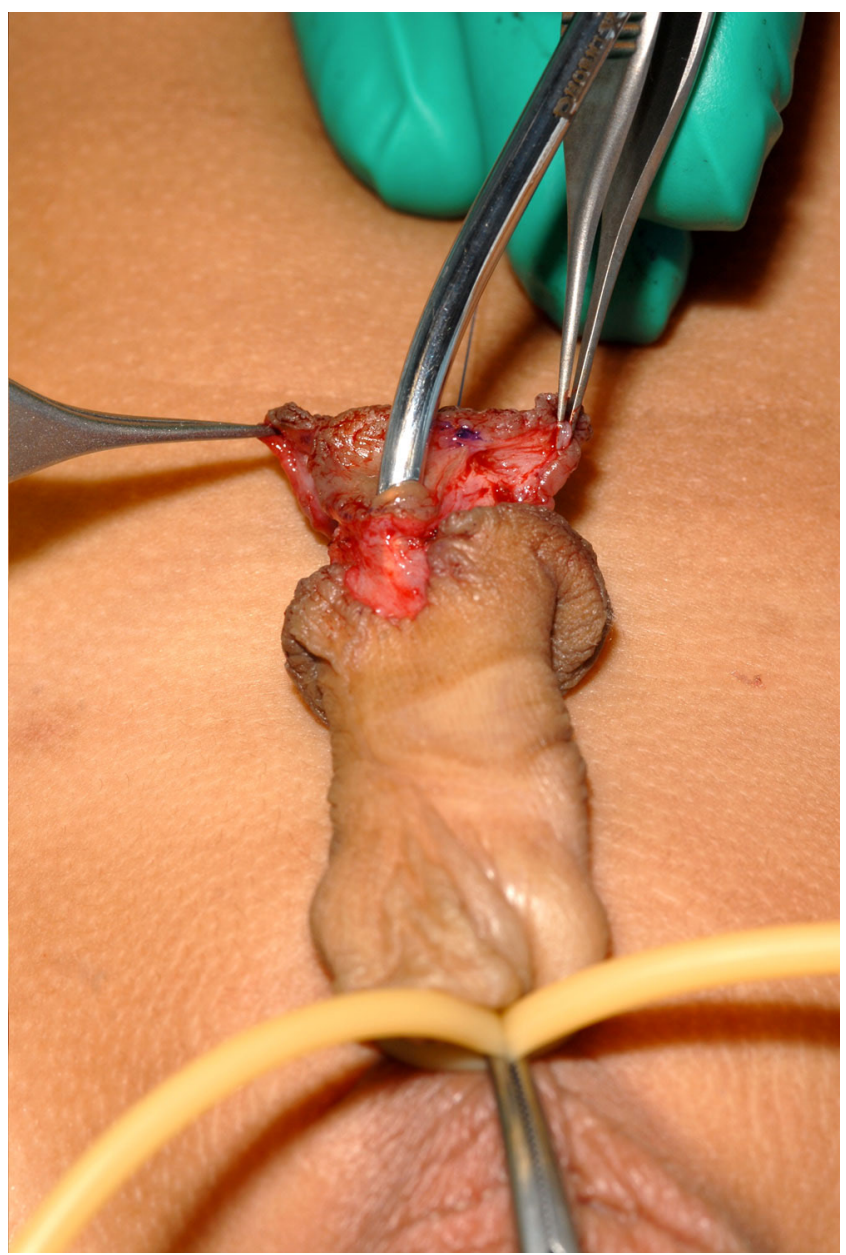

Figure 2: Utilization of a urethral dilator while degloving the ventral skin sufficient to tubularise around the catheter chosen. This varies according to patient age, penis size, and the native urethral plate width; this is left to the clinical judgement of the senior author, hence the steep learning curve associated with hypospadias surgery.

A second layer from the soft tissue surrounding the neourethra is used to reinforce the reconstruction, and help create the deficient corpus spongiosum. It is repaired using the same suture material in a running fashion from proximal to distal. The competency of the neourethra reconstruction is tested with an intraurethral injection of normal saline via a $22 \mathrm{G}$ intravenous cannula (BD Venflon), to delineate possible leakage sites, which if found, are repaired using interrupted sutures. Care is taken to introduce the cannula dorsal to the urethral catheter to avoid injury of the neo-urethra. The previously dissected Dartos flap is transposed ventrally to create a waterproof layer with its distal extent at the level of the coronal sulcus. The Dartos flap is secured with interrupted polyglactin 7-0 sutures (Vicryl-Ethicon). The flap is not used in the distal (glandular and coronal) subset of metal openings, as it would create an unwanted and unnatural bulk under the glans flaps. The glans flaps are brought together with an undyed polyglactin 6-0 suture (Vicryl-Ethicon).

The tourniquet is released at this stage and bleeding vessels are cauterized with bipolar diathermy. After haemostasis is secured the tourniquet is reapplied. The ventral skin is closed in a " $\mathrm{V}-\mathrm{Y}$ " manner starting distally, where the ventral skin is sutured to the glans flap with a four corner rapidly dissolving polyglactin 7-0 suture (Vicryl Rapide-Ethicon) at the frenulum. The remainder of the ventral vertical limb is closed with an interrupted suture using the same material. The excess preputal skin is marked for excision. A number 15 Beaver blade (Beaver-Visitec) is used to make the initial incision and the rest of the excision is carried out with the bipolar diathermy to facilitate a bloodless wound edge, which can be a source of postoperative bleeding in circumcision. Care is taken when closing the subcoronal incision to avoid a rotational deformity. The average time taken for the one stage is between 60-75 min.

\section{Two stage Bracka repair}

The penis is examined under magnification and the morphological findings are processed through the algorithm, at which stage it will indicate that a single stage repair is not suitable. With the two stage approach, there is generally a deficiency of tissues to form a neourethra and coverage.

The glans cleft in the centre of the narrow urethral 
plate is incised vertically with horizontal extensions at the meatus; and glans flaps are raised. A full thickness skin graft is harvested from the inner surface of the dorsally hooded prepuce, its dimensions are not routinely measured, and however, the overall width of the urethral plate and skin graft should be at least $12 \mathrm{~mm}$. The donor is closed primarily using rapidly dissolving polyglactin 7-0 suture (Vicryl RapideEthicon). The skin graft is secured to the defect created using polyglactin 7-0 sutures (Vicryl-Ethicon). The same suture material is used to quilt the skin graft. Polypropylene 6-0 sutures (Prolene-Ethicon) are used to secure the non-adhesive bolster dressing applied to the skin graft. A size $6 \mathrm{Fr}$ silicone Foley's catheter is inserted at this stage. The average time for the first stage is $45 \mathrm{~min}$.

The second stage is performed for the patient 6 months after the first stage, where the graft has healed completely and the tissues have become soft and supple. The second stage is identical to the one stage repair, where by the lateral incisions are performed at the edge of the full thickness skin grafts. The average time taken is similar to the one stage repairs: $60-75 \mathrm{~min}$.

\section{RESULTS}

A total of 102 patients were operated upon between 2009 and 2013. Inclusion criteria included primary hypospadias in the paediatric population. Exclusion criteria, was 1 adult with delayed presentation of primary hypospadias, and 5 cases of phimosis, thought to have concealed hypospadias, treated with circumcision only. Of the 102 patients, 56 underwent a one stage procedure while 46 underwent a two stage procedure. Mean age at presentation was 22 weeks \pm 29.75 (SD). The mean age at surgery was 25 months \pm 11.56 (SD).

Of the 102 cases, $41.2 \%(n=42)$ were subcoronal, $20.6 \%(n=21)$ coronal, $13.7 \%(n=14)$ glandular, $1 \%$ $(n=1)$ terminal, $11.8 \%(n=12)$ distal shaft, $2.9 \%(n=$ 3) mid-shaft, $3.9 \%(n=4)$ proximal shaft, and $4.9 \%(n$ =5) penoscrotal [Figure 3].

Early complications were those encountered within 6 weeks of the operative procedure, whilst any complication encountered after that period would be classed as a late complication. Early complication rate was $4.9 \%(n=5)$ and they included dislodged/snapped catheters $1.9 \%(n=2)$, wound dehiscence and infection $2.9 \%(n=3)$. Overall late complication rate was $10.7 \%$ $(n=11)$. The most common complication was urethrocutaneous fistula occurring in $8.8 \%(n=9)$. The fistula rate specific to one stage and two stage procedures was $5.3 \%(n=3)$ and $13 \%(n=6)$ respectively. Other late complications included, altered urine stream $0.9 \%$ $(n=1)$ and urethral stricture $0.9 \%(n=1)$ [Figure 4]. No complications related to bleeding were encountered. Re-operation was required for 2 patients with wound dehiscence and 9 patients with fistula.

\section{DISCUSSION}

Many techniques and modifications have been described for the repair of hypospadias, yet there has been no general consensus on a specific procedure for a given subset of hypospadias meatal positions or morphology. Prat et al. ${ }^{[3]}$ published a series that covered three decades including 820 patients, highlighting different techniques and overall results. They performed meatal advancement and glanduloplasty (MAGPI), TIP, two stage procedures, onlay tabularised island flap, yet with a high incidence of complications. We employ the use of a simple double-armed algorithm ending in two procedures, which we feel are adequate to address treatment of hypospadias.

The algorithm [Figure 5] disregards the meatal position, and addresses other morphological findings; chordee, adequacy of the urethral plate, glans cleft, and presence of a urethral bar. A similar algorithm was utilised by Coleman et al., ${ }^{[4]}$ whereby they used

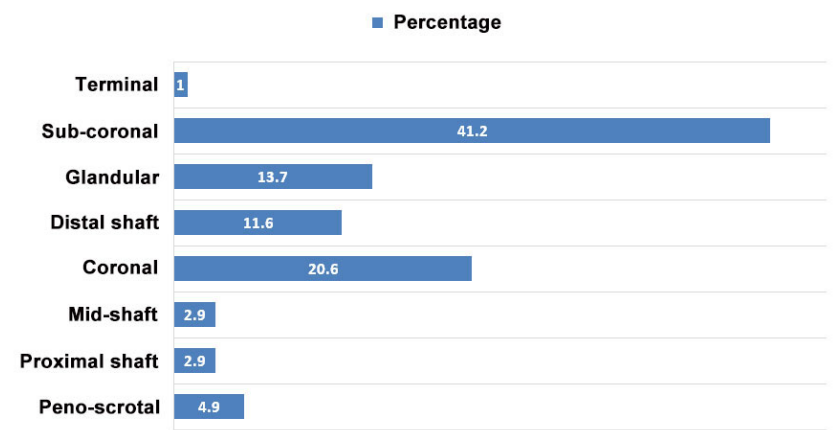

Figure 3: Hypospadias subtypes (\%)

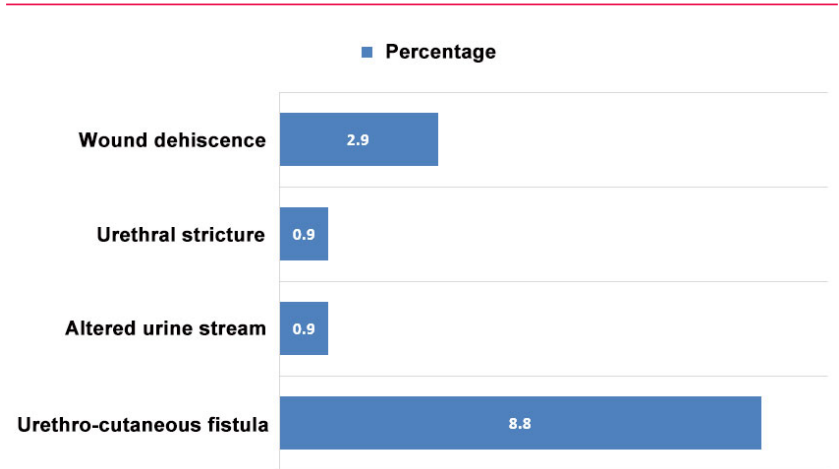

Figure 4: Complication rate (\%) 
their algorithm to arrive at a choice of a one or two stage repair.

Chordee was present in $94.1 \%(n=96)$ of our patients with varying degrees; mild $81 \%(n=78)$, moderate $7 \%(n=7)$, severe $12 \%(n=11)$. In the presence of mild to moderate chordee and adequate urethral plate, adequate glans cleft, and a thin or no horizontal bar within the urethral plate, a one stage repair (Snodgrass type without urethral plate incision) is used. This procedure was initially described as TIP. $^{[5]}$ We found no need to incise the urethral plate in all but 1 patient.

Adequacy of the urethral plate is based on the fact that it will be tubularised around a 6 Fr urinary catheter, as is standard in our practice. A urethral plate of $8-10 \mathrm{~mm}$ or more is sufficient for tubularisation without the need for augmentation. If the findings are not in favour of a one stage procedure as per algorithm, then a two stage Bracka repair is used.

The microscope was used for all the hypospadias repairs in our series. We found that its use was quite ergonomic, when surgeons were sat in an upright posture with relaxed shoulders and elbows rested on the operative table; this is important for surgeons who will be doing hypospadias surgery regularly. The microscope used also had a separate video screen; this displayed a view of the operative field as seen by the surgeons. This is an invaluable teaching aid, as often there may be a second assistant that will not be able to see the filed in the absence of this screen. Not only did the microscope serve an ergonomic and teaching purpose, but also provided magnification and

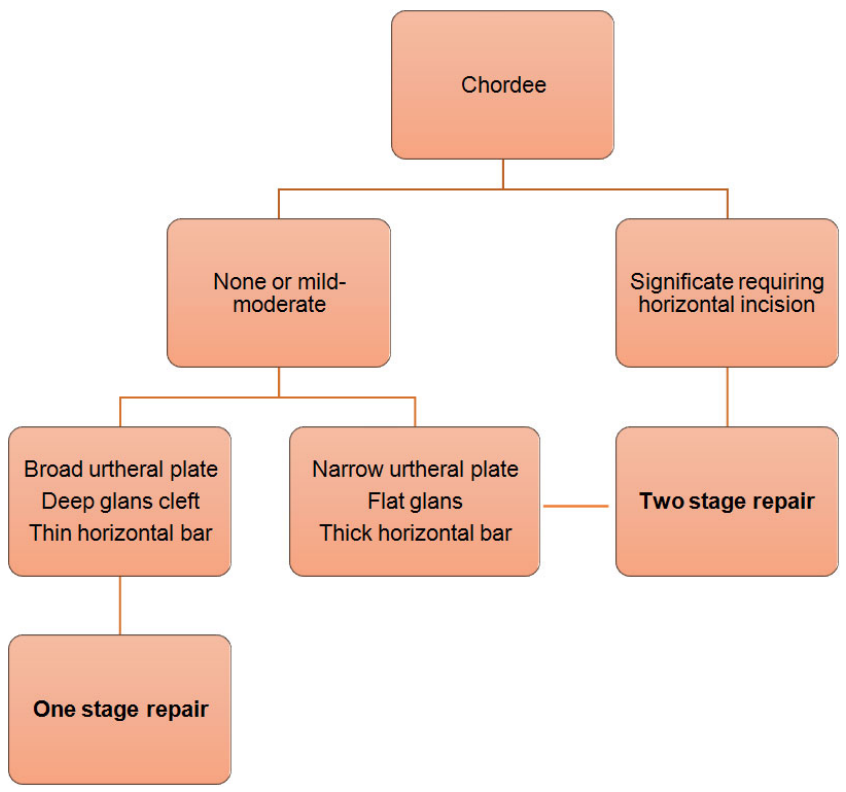

Figure 5: Algorithm for choice of repair illumination required for surgery on delicate and small structures, where along with appropriate microsurgical instruments are hallmark to a meticulous repair. Wacksman reported that the use of the microscope has allowed for the use of small fine sutures with great accuracy translating into results..$^{[6]}$ Wesson et al. ${ }^{[7]}$ found that it was a very useful teaching aid for residents. Unscrubbed junior colleagues observing will also be able to chronologically follow the surgical steps, to better understand this type of surgery, which does have a steep learning curve. It is also of note that other healthcare staffs in theatre are aware of the nature of the surgery that is being performed, and to able to identify what stage of the surgery has been reached at any given time.

Gilbert et al. ${ }^{[8]}$ demonstrated that they experienced fewer complications when using the microscope. The non-microscope group had 17 complications, 12 of which required reoperation (24\%), while the microscope group had 8 complications of which 4 required reoperation (6.5\%). A head mounted microscope has been utilized in hypospadias surgery, again noticing a decrease in complications. ${ }^{[9]}$ Although this may provide magnification, there is no evidence to prove that it is more ergonomic than the surgical microscope.

In the 102 patients in our case series, 56 had one stage repairs while 46 had two stage Bracka repairs. Postoperative edema was not observed in any of our cases. Bhat and Mandal ${ }^{[10]}$ found that edema was the second most common early complication after hypospadias surgery. Urethro-cutaneous fistula remains the most common complication encountered by all. Snodgrass reported fistula rates at $13 \%$ in mid-shaft hypospadias and up to $37 \%$ in proximal shaft, depending on single or two layer closure and whether polyglactin or chromic was used. ${ }^{[11]}$ The two stage procedure was popularized by Bracka, ${ }^{[12]}$ and reports of a gross fistula rate of $5.7 \%$ and a stricture rate of $7 \%$. It is worthwhile mentioning that there was no mention of magnification in the Snodgrass series, whereas Bracka does not rely on magnification at all. ${ }^{[11,12]}$ In our series we experienced one stricture and total fistula rate of $8.8 \%, 5.3 \%$ and $13 \%$ for one stage and two stage repairs respectively. The stricture was picked up by urethroscopy. Our postoperative follow-up regimen includes review at 1 and 2 weeks postoperatively. They then are reviewed at 3 months, and from then on yearly till 5-6 years of age before discharge. Khan et al. ${ }^{[13]}$ reported an increase in complications as a result of lack of magnification and microsurgical instruments. Hypospadias surgery has as steep learning curve, and it is more likely that more complications will be encountered earlier on in

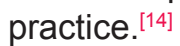


In conclusion, hypospadias surgery is practiced by a few surgical sub-specialties. It has been practiced for a long time and has evolved remarkably. There is a plethora of techniques and complication rates are still considered relatively high. Strategically approaching hypospadias with this simple algorithm and utilizing the microsurgical instruments with a surgical microscope will help in performing meticulous repairs. This approach will be much appreciated by those embarking in a career of hypospadiology.

\section{Authors' contributions}

Manuscript's preparation: M. Maher, M. Chasapi

Manuscript's review: M. Dalal

Concept design: M. Dalal

Literature search: M. Chasapi, M. Maher

\section{Financial support and sponsorship}

None.

\section{Conflicts of interest}

There are no conflicts of interest.

\section{Patient consent}

All patients have signed a formal informed consent form for medical photography allowing publication.

\section{Ethics approval}

All procedures performed are in accordance with departmental ethics standards.

\section{REFERENCES}

1. Djakovic N, Nyarangi-Dix J, Ozturk A, Hohenfellner M. Hypospadias. Adv Urol 2008;2008:501-35.

2. Manzoni G, Bracka A, Palminteri E, Marrocco G. Hypospadias surgery: when, what, by whom? BJU Int 2004;94:188-95.

3. Prat D, Natasha A, Polak A, Koulikov D, Prat O, Zilberman M, Abu Arafeh W, Moriel EZ, Shenfeld OZ, Mor Y, Farkas A, Chertin B. Surgical outcomes of different types of hypospadias repair during three decades in a single center. Urology 2012;79:1350-3.

4. Johnson D, Coleman DJ. The selective use of a single-stage and a two-stage technique for hypospadias correction in 157 consecutive with the aim of normal appearance and function. Br J Plast Surg 1998;51:195-201.

5. Snodgrass W, Koyle M, Manzoni G, Hurwitz R, Caldamone A, Ehrlich R. Tubularized incised plate hypospadias repair: results of a multicenter experience. J Urol 1996;156:839-41.

6. Wacksman J. Repair of hypospadias using new mouth controlled microscope. Urology 1987;29:276-8.

7. Wesson L, Mandell J. Single-stage hypospadias repair using the operating microscope. Microsurgery 1985;6:182-4.

8. Gilbert DA, Devine CJ Jr, Winslow BH, Horton CE, Getz SE. Microsurgical hypospadias repair. Plast Reconstr Surg 1986;77:460-7.

9. Chiummariello S, Arleo S, Rizo I, Monarca C, Dessy LA, Scuderi N Alfano C. New head-mounted miniaturized microscope in hypospadia surgical correction. Minerva Chir 2013;68:207-12.

10. Bhat A, Mandal AK. Acute post-operative complications of hypospadias repair. Indian J Urol 2008;24:241-8.

11. Snodgrass W, Yucel S. Tubularized incised plate for mid-shaft and proximal hypospadias repair. J Urol 2007;177:698-702.

12. Bracka A. A versatile two-stage hypospadias repair. Br J Plast Surg 1995; 48:345-52

13. Khan M, Majeed A, Hayat W, Ullah H, Naz S, Shah S, Tahmeed T, Yousaf K, Tahir M. Hypospadias repair: a single center experience. Plast Surg Int 2014;2014:453039.

14. Titley OG, Bracka A. A 5-year audit of trainees experience and outcomes of hypsospadias surgery. Br J Plast Surg 1998;51:370-5. 